\title{
Excitation Intensity- and Temperature-Dependent Photoluminescence Study of InAs/GaAs Sub-monolayer-Quantum Dot
}

\author{
Minseak Kim ${ }^{\mathrm{a}, \#}$, Hyun Jun Jo, ${ }^{\mathrm{a}, \sharp}$, Yeongho Kim ${ }^{\mathrm{b}}$, Seung Hyun Lee ${ }^{\mathrm{c}}$, Sang Jun Lee ${ }^{\mathrm{b}}$, \\ Christiana B. Honsberg ${ }^{\mathrm{d}}$, and Jong Su Kim ${ }^{\mathrm{a}, *}$ \\ ${ }^{a}$ Department of Physics, Yeungnam University, Gyeongsan, Korea \\ ${ }^{b}$ Korea Research Institute of Standards and Science, Daejeon, Korea \\ ${ }^{c}$ Department of Electrical and Computer Engineering, Ohio State University, Columbus, USA \\ ${ }^{d}$ School of Electrical, Computer and Energy Engineering, Arizona State University, Tempe, USA
}

Received July 16, 2018; revised September 20, 2018; accepted September 21, 2018

\begin{abstract}
Optical properties of InAs/GaAs submonolayer-quantum dot (SML-QD) have been investigated using excitation intensity $\left(I_{e x}\right)$ - and temperature-dependent photoluminescence $(\mathrm{PL})$. At a low temperature $(13 \mathrm{~K})$ strong PL was observed at $1.420 \mathrm{eV}$ with a very narrow full-width at half maximum, of $7.09 \mathrm{meV}$. The results of the $I_{e x}$ dependence show that the PL intensities increase with increasing $I_{e x}$. The enhancement factors $(k)$ of PL increment as a function of $I_{e x}$ are 3.3 and 1.22 at low and high $I_{e x}$ regime, respectively. The high $k$ value at low $I_{e x}$, implies that the activation energy of the SML-QDs is low. The calculated activation energy of the SML-QDs from temperature dependence is 30 $\mathrm{meV}$.
\end{abstract}

Keywords: PL, SML QDs, Photoluminescence, Activation enery

\section{Introduction}

Owing to their high absorbing capability near the infrared (IR) wavelength regime, InAs/GaAs quantum dots (QDs) are considered as suitable candidates for fabrication of high-efficiency intermediate-band solar cells [1-4].

InAs/GaAs QDs are mostly formed by StranskiKrastanov (SK) mode [5]. However, in S-K growth mothod, strain-related defects caused by interface strain between InAs and GaAs are unavoidable. Defects act as carrier trapping and recombination centers, resulting in degradation of the optical properties and current transport performance [6,7]. In S-K method of growing QDs, a wetting layer (WL) is formed and electrons are missed into a wetting layer rather than a quantum dot. This problem reduces the open-circuit voltage of the solar cell. For these reasons, it is important to suppress a defect generation and to maintain the crystal quality of QDs without WL formation to achieve high performance of devices. Recently, in attempts to fabricate defect-free QDs, InAs QDs with low defect density caused by strain, submonolayer (SML) deposition method has been suggested

${ }^{\#}$ Contributed equally to this work as first author.

*Corresponding author

E-mail: jongsukim@ynu.ac.kr as an alternative method for the SK growth mode [8-10]. In principle, the SML growth process consists of InAs SML of thickness less than 1.0 monolayer (ML), while conventional InAs SK-QDs are typically formed by depositing over 1.7 ML (critical thickness in S-K mode) of InAs on the lattice-mismatched GaAs surface $[9,11,12]$. Since the size of quantum dots formed in the SML-QD method is smaller than that of the S-K method, the carrier escape efficiency is improved and carrier transportation is facilitated [13].

In this study, we investigated optical properties of InAs/ GaAs SML-QD using photoluminescence (PL) with various excitation intensities and temperatures.

\section{Experimental details}

Figure 1 shows a schematic sample structure of the InAs/ GaAs SML-QD samples used in this study. Samples were grown on a semi-insulating (SI) GaAs (100) substrate by a solid source molecular beam epitaxy (MBE) system. An undoped-GaAs buffer layer of thickness $400 \mathrm{~nm}$ was grown on the SI-GaAs substrate.

To form a SML-QD, a $0.5 \mathrm{ML}$ thick InAs layer was grown on the GaAs buffer layer and then $2.5 \mathrm{ML}$ thick GaAs layer was employed to cap the InAs layer, this sequence was repeated 5 times to form a SML-QD. After 


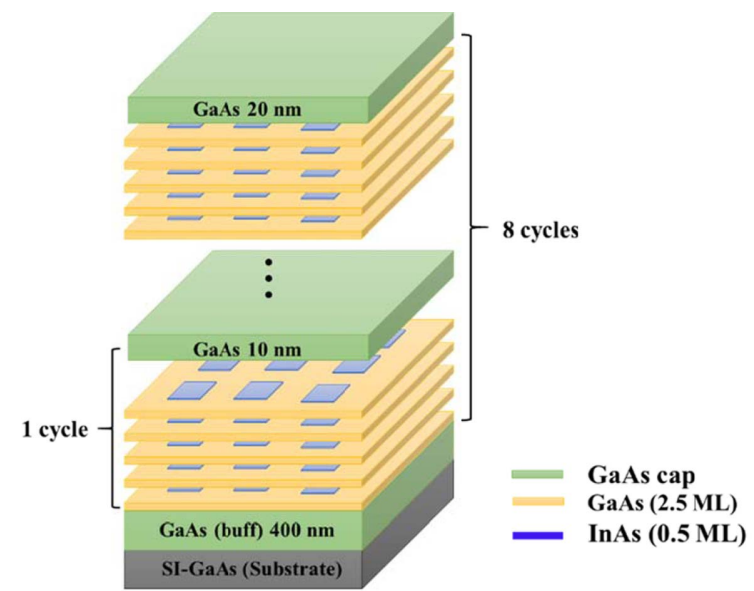

Figure 1. Schematic structure of InAs/GaAs SML-QDs.

formation of the SML-QD, $10 \mathrm{~nm}$-thick GaAs layer was used as a spacer layer. 8 periods of SML-QD layers were formed. Each period of the SML-QD layer consists of 5 cycles of InAs $(0.5 \mathrm{ML}) / \mathrm{GaAs}(2.5 \mathrm{ML})$ and is covered with a $10 \mathrm{~nm}$-thick GaAs layer.

For PL experiment, a laser diode $(637 \mathrm{~nm})$ was used as an excitation light source with its light incident on the sample surface. The excitation intensity $(21.6 \sim 886 \mathrm{~mW} /$ $\mathrm{cm}^{2}$ ) was controlled using a neutral density filter. The PL intensity as a function of emission wavelength was measured using a Si detector.

\section{Results and discussion}

Figure 2 shows low temperature PL spectra of InAs/ GaAs measured at a temperature of $13 \mathrm{~K}$ under excitation intensity $\left(I_{e x}\right)$ of $8.3 \mathrm{~mW} / \mathrm{cm}^{2}$. The PL peak is observed at $1.420 \mathrm{eV}$ for InAs/GaAs SML-QD with the $7.09 \mathrm{meV}$ fullwidth at half maximum (FWHM) as shown in the inset. Han et. al. reported $1.7 \mathrm{ML}$ S-K QD has $\sim 50 \mathrm{meV}$ of FWHM in the change of FWHM of PL spectra for InAs QD size distribution [2]. In this study, the SML-QD has significantly narrow FWHM, which implies that the SMLQD has high uniformity of size.

To investigate the effect of $I_{e x}$ on the properties such as PL intensity, peak shift, and FWHM, on SML-QDs, $I_{e x}$ dependent PL was performed with various intensities $(8.3$ $\sim 1,666 \mathrm{~mW} / \mathrm{cm}^{2}$ ) of incident light at $\mathrm{T}=13 \mathrm{~K}$ and the results are shown in Fig. (a)-(d). Figure 3(a) illustrates that the PL peak shifts to lower energy for $I_{e x}>410 \mathrm{~mW} / \mathrm{cm}^{2}$ and the PL intensity gradually saturates as the value of $I_{e x}$ increases. The position and FWHM of the PL peak as a function of the $I_{e x}$ are shown in Fig. 3(b) and 3(c) to confirm the above. A little red-shift of $\sim 0.74 \mathrm{meV}$ is due to the increase in recombination of relatively larger QDs and low energy QDs, by the process of escape and re-trapping of the excess carriers inside the QDs. The value of FWHM remains constant for a considerable intensity regions. The constant FWHM for a large intensity region implies that

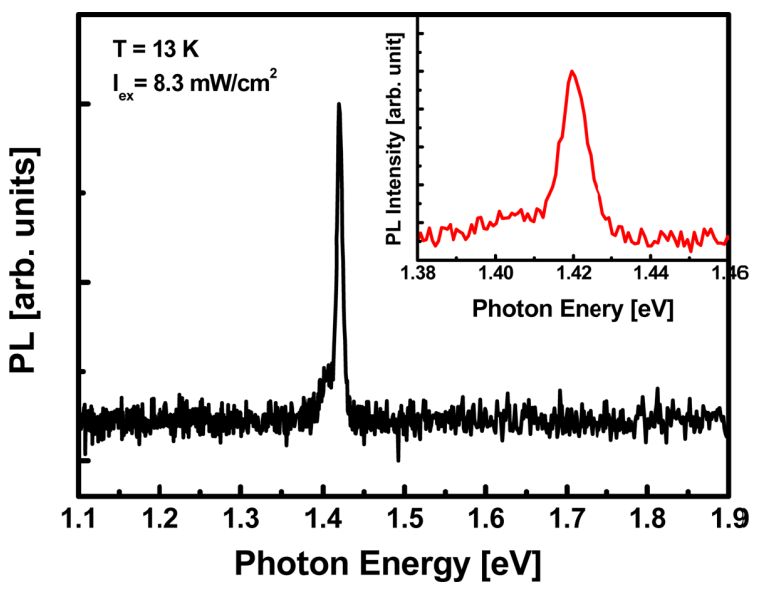

Figure 2. PL spectra of InAs/GaAs SML-QD at $13 \mathrm{~K}$.
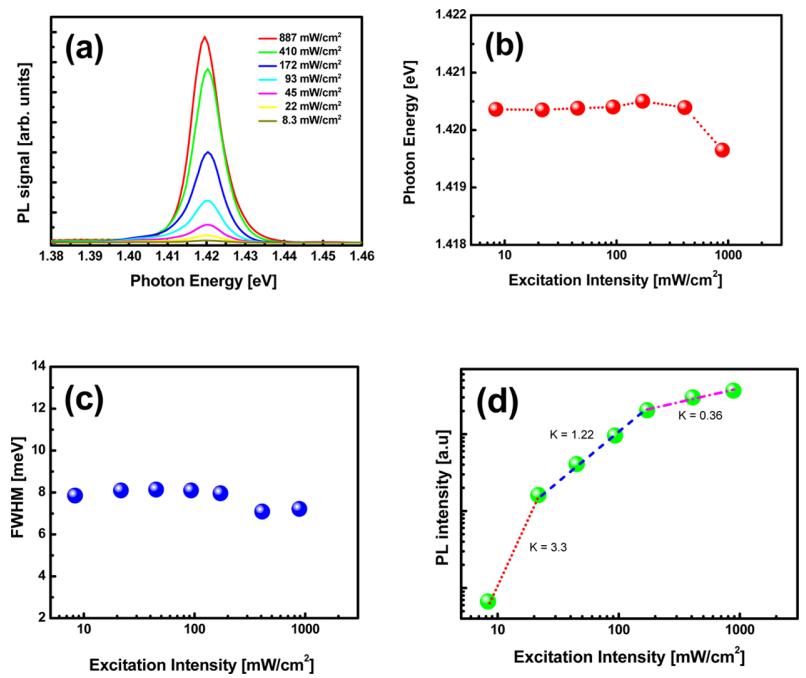

Figure 3. (a) PL spectra with various excitation intensity, (b) excitation intensity-dependent PL peak shift, (c) excitation intensity-dependent FWHM of PL spectra and (d) PL intensity as a function of excitation intensity of InAs/GaAs SML-QD at $T=13 \mathrm{~K}$.

the SML-QD has high uniformity. In the PL spectra, the emission intensity increases with $I_{e x}$, and the slope of the emission intensity-curve depends on the origin of the emission [14]. The intensity of a spontaneous emission by an intrinsic transition, such as the interband and the exciton transitions at the PL spectra, is proportional to the natural logarithm of $I_{e x}$ when the origin of emission is operating independently and there is no reciprocal movement of the carrier. Figure 3(d) depicts the intensities of the peak as a function of $I_{e x}$ at $\mathrm{T}=13 \mathrm{~K}$. The relationship between $I_{e x}$ and the emission intensity in the PL signal can be expressed as follows [14].

$$
k=\frac{\log I_{P L}}{\log I_{e x}}
$$

where, $k$ is the emission coefficient, $I_{P L}$ is the emission intensity, and $I_{e x}$ is the excitation intensity. Emission related to the exciton gave $1<k<2$ for the excitation intensity 

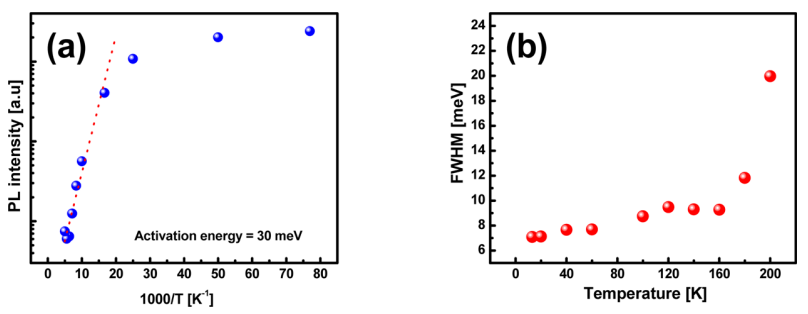

Figure 4. (a) PL intensity as a function of reciprocal temperature and (b) temperature-dependent FWHM of the PL spectra.

dependence. In addition, the emission related to limited energy states gave $k<1$. In the intensity region below $22 \mathrm{~mW} / \mathrm{cm}^{2}$, the value of $k$ is 3.3 . In the region from 22 to $172 \mathrm{~mW} / \mathrm{cm}^{2}$, the value of $k$ is 1.22 , similar to that for an exciton transition. In the region above $172 \mathrm{~mW} / \mathrm{cm}^{2}$, the value of $k$ is 0.36 , which is similar to that for a defect transition. Generally, the QDs with high barrier height, in which case the size of the QDs is large, gave $k=1$ [15]. However, in the case of QDs with low barrier height, slope of $k$ is not linear but exhibits a superlinear dependence.

At lower $I_{e x}$, when the escape of the carrier inside the QDs is easy, the value of $\mathrm{k}$ is greater than 1 because the escaped carriers do not contribute to emission of the QDs as the phenomena of escape and re-trapping of the electron and hole are independent [15]. However, this superlinearity becomes weaker with increasing $I_{e x}$ and approaches linearity (slope of 1) at the high intensities. With further increase in the value of $I_{e x}$, the states of the SML-QDs are easily filled because the sizes are smaller than general QDs grown by S-K method. Hence, the carriers, which are confined to the QDs, can escape easily and move to other states. Therefore, the recombination rate decreases inside the QDs thus decreasing the value of $k$. In the case of high $I_{e x}$, most carriers escape, and the recombination rate inside the QDs is further reduced.

To confirm our assumption, that the barrier height of the SML-QDs is low,s the temperature-dependent PL measurements were performed. The integrated PL intensity as a function of reciprocal of temperature is depicted in Fig. 4(a). The measurements were performed with a laser excitation power of $887 \mathrm{~mW} / \mathrm{cm}^{2}$. The activation energy of the QDs can be determined from the PL signal, as shown in Fig. 4(a). The change in the integrated PL intensity with temperature is given as follows [15].

$$
I=\frac{I_{0}}{1+C \exp -E_{a} /\left(k_{B} T\right)}
$$

where, $I_{0}$ is the intensity at $\mathrm{T}=0 \mathrm{~K} . E_{a}$ and $k_{B}$ are the binding energy and Boltzmann constant, respectively. $C$ is proportionality constant. As expected, the activation energy of the SML-QDs from the fit was $30 \mathrm{meV}$. This value of $30 \mathrm{meV}$ is sufficiently smaller than the value of $225 \mathrm{meV}$ of the S-K QDs [16]. This lower activation energy value of the SML-QDs promotes photo-generated carrier escape.
The temperature-dependent FWHM of the PL spectra is depicted in Fig. 4(b). The value of FWHM increases with increase in $I_{e x}$ value because the escaped carriers can contribute to the emission of smaller or larger QDs into the surroundings. The rapid increase the FWHM at T $=160 \mathrm{~K}$ is due to increase in escape by the low activation energy.

\section{Conclusions}

$I_{e x}$ and temperature-dependent PL spectra of InAs/GaAs SML-QD have been investigated. At low temperature (13 $\mathrm{K})$ strong PL was observed at $1.420 \mathrm{eV}$ with very narrow FWHM of $7.09 \mathrm{meV}$. The result of the $I_{e x}$ dependence showed that the PL intensities increased and finally saturated with increasing $I_{e x}$. The value 3.3 of $k$, below 22 $\mathrm{mW} / \mathrm{cm}^{2}$ intensity is reduced at higher intensities due to a decrease in the escape and re-trapping effects. In the intensity region above $172 \mathrm{~mW} / \mathrm{cm}^{2}$, the states of the SML-QDs are easily filled, so most carriers escape, and the recombination rate inside the QDs is further reduced. The activation energy of the SML-QDs obtained from the temperature dependence was $30 \mathrm{meV}$. This lower activation energy of the SML-QDs aids the escape of the photogenerated carriers.

\section{Acknowledgments}

This research was supported by Basic Science Research Program through the National Research Foundation of Korea (NRF) funded by the Ministry of Education (NRF2017R1A6A3A11028070). This work was supported by the National Research Foundation of Korea (NRF) grant funded by the Korea government (NRF-2018R1D1A3B 07050824).

\section{References}

[1] K. A. Sablon, J. W. Little, V. Mitin, A. Sergeev, N. Vagidov, and K. Reinhardt, Nano. Lett. 11 (2011).

[2] I. S. Han, R. P. Smith, J. S. Kim, S. K. Noh, S. J. Lee, C. L. Lee, and J.-Y. Leem, Sol. Energy Mater. Sol. Cells 155 (2016).

[3] A. Martí, E. Antolín, E. Cánovas, N. López, and A. Luque, Proceedings of the 21st European Photovoltaic Solar Energy Conference, (Dresden, Germany, Sept. 4-8, 2006), pp. 99-102.

[4] A. Nasr, Optics \& Laser Technology, 48 (2013). View at Google Scholar.

[5] P. B. Joyce, T. J. Krzyzewski, G. R. Bell, B. A. Joyce, and T. S. Jones, Phys. Rev. B 58 (1998).

[6] S. M. Willis, J. A. R. Dimmock, F. Tutu, H. Y. Liu, M. G. Peinado, H. E. Assender, A. A. R. Watt, and I. R. Sellers, Sol. Energy Mater. Sol. Cells 102 (2012).

[7] J. Wu, Q. Jiang, S. Chen, M. Tang, Y. I. Mazur, Y. Maidaniuk, M. Benamara, M. P. Semtsiv, W. T. Masselink, K. A. Sablon, G. J. Salamo, and H. Liu, ACS Photonics 3 (2016).

[8] S. Krishna, D. Zhu, J. Xu, K. K. Linder, O. Qasaimeh, P. Bhattacharya, and D. L. Huffaker, J. Appl. Phys. 86 (1999).

[9] Z. Xu, D. Birkedal, J. M. Hvam, Z. Zhao, Y.Liu, K. Yang, A. Kanjilal, and J. Sadowski, Appl. Phys. Lett. 82 (2003).

[10] T. D. Germann, A. Strittmatter, J. Pohl, U. W. Pohl, D. Bimberg, J. Rautiainen, M. Guina, and O. G. Okhotnikov, Appl. Phys. Lett. 92 (2008). 
112 Minseak Kim, Hyun Jun Jo, Yeongho Kim, Seung Hyun Lee, Sang Jun Lee, Christiana B. Honsberg, and Jong Su Kim

[11] I. L. Kresnikov, N. N. Ledentsov, A. Hoffmann, and D. Bimberg, Phys. Stat. Sol. (a) 183 (2001).

[12] N. N. Ledentsov and D. Bimberg, J. Crystal Growth 255 (2003).

[13] Y. Kim, K.-Y. Ban, and C. B. Honsberg, Appl. Phys. Lett. 106 (2015).
[14] T. Schmidt, K. Lischka, and W. Zulehner, Phys. Rev. B 45 (1992).

[15] E. C. Le Ru, J. Fack, and R. Murray, Phys. Rev. B, 67 (2003).

[16] K. S. Lee, G. Oh, E. K. Kim, and J. D. Song, Applied Science and Convergence Technology 26 (2017) 